\title{
A Comparison of Evidence Fusion Rules for Situation Recognition in Sensor-Based Environments
}

\author{
Susan McKeever \\ Technological University Dublin, susan.mckeever@tudublin.ie \\ Juan Ye \\ University of St. Andrews
}

Follow this and additional works at: https://arrow.tudublin.ie/scschcomcon

Part of the Artificial Intelligence and Robotics Commons, and the Theory and Algorithms Commons

\section{Recommended Citation}

McKeever, S., \& Ye, J. (2013). A Comparison of Evidence Fusion Rules for Situation Recognition in SensorBased Environments. AML 2013: Evolving Ambient Intelligence:Workshops, Dublin, Ireland, 3-5, December doi:10.1007/978-3-319-04406-4_16

This Conference Paper is brought to you for free and open access by the School of Computer Sciences at ARROW@TU Dublin. It has been accepted for inclusion in Conference papers by an authorized administrator of ARROW@TU Dublin. For more information, please contact arrow.admin@tudublin.ie, aisling.coyne@tudublin.ie,gerard.connolly@tudublin.ie.

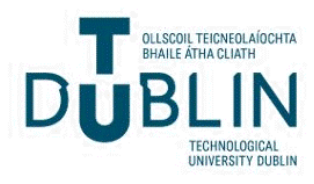




\title{
A comparison of evidence fusion rules for situation recognition in sensor-based environments
}

\author{
Susan McKeever ${ }^{1}$, Juan $\mathrm{Ye}^{2}$
}

\begin{abstract}
Dempster-Shafer (DS) theory, and its associated Dempster rule of combination, has been widely used to determine belief based on uncertain evidence sources. Variations to the original Dempster rule of combination have appeared in the literature to support particular scenarios where unreliable results may result from the use of original DS theory. While theoretical explanations of the rule variations are explained, there is a lack of empirical comparisons of the DS theory and its variations against real data sets. In this work, we examine several variations to DS theory. Using two real-world sensor data sets, we compare the performance of DS theory and several of its variations in recognising situations. The empirical results shed insight on how to select these fusion rules based on the nature of sensor data, the relationship of this data over time to the higher level hypotheses and the choice of frame of discernment.
\end{abstract}

Key words Evidence theory, Dempster Shafer theory, situations, situation recognition, uncertainty, uncertain reasoning

\section{Introduction}

Situations are human understandable representations of the environment that are of interest to a pervasive application. In the context of smart-homes, the term situation is often used interchangeably with activities, where the ambient system detects situation such as 'taking a shower' or 'preparing breakfast'. This task of situation recognition is a critical, continuous process in ambient environments, such as those monitoring the health of elderly patients in their homes.

Sensor data is inherently unreliable, noisy, prone to delay and imprecise. i.e. it suffers from uncertainty. For pervasive systems which rely on sensor data, the input such as a location tag reading is ambiguous [1], so inference from sensor data cannot be treated as fact, but simply evidence of fact. Users' actions can contribute to degradation of information quality, such as the failure of users to carry their locator tags [2]. Uncertainty of sensor information should be tracked and preserved to determine uncertainty at higher levels of context. Furthermore, the process of interpretation of sensed context is also subject to ambiguity and approximation. Some context concepts are fuzzy, so are subject to imprecision, such as the concepts of 'near' or 'warm'. Infer-

\footnotetext{
${ }^{1}$ School of Computing, Dublin Institute of Technology, Ireland

${ }^{2}$ School of Computer Science, University of St. Andrews, United Kingdom
} 
ence rules are not constant, such as a user 'sometimes' using a microwave when engaged in 'preparing breakfast'. In our previous work [3], we have applied Dempster Shafer theory to quantify and accommodate this uncertainty in order to maximize our situation recognition results.

Dempster-Shafer theory is a theory of uncertain reasoning that is widely used in domains where information (evidence) is known to be imperfect and reasoning uncertain, such as medical diagnosis, quality control and process engineering. DS theory, and its associated Dempter rule of combination, has been widely used to determine belief based on uncertain evidence sources. In ambient environments, DS theory has been applied to situation recognition, based on sensors embedded in the environment [4] [5] [3]. The use of DS theory removes the reliance of training data required by learning approaches, such as Bayesian networks, Naive Bayes, Hidden Markhov models, and Decision Trees.

The field of evidence theory has widened beyond the original Dempster-Shafer theory, with variations to the theory proposed by other practitioners. Variations to the the original Dempster rule of combination from [6] [7] [8] [9] have been proposed in order to support particular scenarios where unreliable results may result from the use of original DS theory. While theoretical explanations of the rule variations appear in the literature, there is a lack of empirical comparisons of DS theory and its variations against real data sets. In this work, we examine several variations to DS theory. Using two sensor data sets, we compare the performance of DS theory and these variations in recognising situations from the sensor data. By comparing the results, we emphasise the nature of sensor data as a selection factor in the fusion rule, the impact of temporal spread of sensor data and the impact of multiple hypotheses in the frame of discernment.

The rest of the paper is organised as follows. Section 2 overviews the research in activity recognition and focus on the application of Dempster-Shafter theory in the area. Section 3 introduces the background of Dempster-Shafter theory and its alternative fusion rules, which we evaluate and compare on two independent data sets (described in Section 4) in Section 5. The paper concludes in Section 6.

\section{Related Work}

The ability to recognise higher level situations or context is an ongoing area of research in the pervasive systems field. Various machine learning approaches, have been applied to issue of uncertain sensor data environments. Naives Bayes classifiers have been used by Korpippa [10] and Tapia et al [11]. Ranganathan et al [12] , Gu [13] have applied Bayesian networks. Hidden Markov Models which considers the sequence of sensor data triggers have been applied by Clarkson et al [14], Choujaa et al [15] and van Kasteren [16]. The use of these approaches requires training data which can be problematic to collect and annotate in a sensored environment. 
Dempster-Shafer theory has recently received greater attention in the domain of context-aware systems due to its lack of reliance on training data and its ability to cater for uncertainty in the reasoning process. The first use of the theory for context-aware systems was Wu's approach [17] [18] to fuse sensor data into higher level contexts. Wu's main contribution is the definition of a dynamic discount factor for sensors that changes over time.

Hong et al. [19] [20] apply Dempster-Shafer theory to define an evidence based activity (situation) model that uses sensor data for activity recognition in a smart home. Hong expands on Wu's work by using evidence propagation to bring evidence up through a hierarchy, so that activities can be recognised, as opposed to just abstracted contexts. Zhang et al. [5] use Dempster-Shafer theory for reasoning about activities. Their work focuses on resolving computation intensiveness of evidence fusion and addressing Zadeh's paradox [21], whereby conflicting evidence sources can give paradoxical results by granting majority belief to a minority opinion. In our previous work, we extended DS theory to allow for temporal knowledge to be incorporated into the evidential reasoning process [3].

DS theory has been extended by [7] [6] [8] [20] to allow for modifications to rules, in order to address particular limitations in the original DS rule of combination. In this paper, we examine variations to DS theory, and compare their performance for situation recognition on two sensor data sets. The purpose is to do an empirical comparison of DS theory and its variations, thus highlighting the subtleties of evidential rules when applied to real world sensor data. This will bolster existing theoretical work already done in this field with real examples.

\section{Dempster Shafer theory and alternative fusion rules}

\subsection{Dempster Shafer theory}

DS theory [9] is a theory of uncertain reasoning that is widely used in domains where information (evidence) is known to be imperfect and uncertain. In a DS theory reasoning scheme, the set of possible hypotheses are collectively called the frame of discernment. This frame' $\Omega$ represents the set of choices $\left\{h_{1}, h_{2}, \ldots h_{\mathrm{n}}\right\}$ available to the reasoning scheme, where sources (such as sensors) assign belief or evidence across the hypotheses in the frame. Hypotheses can be any subsets of the frame, ranging from singletons in the frame to combinations of elements in the frame. For example, a calendar sensor that monitors whether a user is scheduled to be in a meeting or not assigns belief across a frame of discernment that includes hypotheses \{meeting, coffee break, busy at desk \}. When the calendar indicates that the user does not have a meeting, belief is assigned by the calendar sensor to 'not meeting' situations i.e. the combination of \{coffee break, busy at desk\}. It assigns zero belief to \{meeting\}. For- 
mally, $2^{\Omega}$ denote the set of all subsets of $\boldsymbol{\Omega}$ to which a source of evidence can apply its belief. The function $m: 2^{\Omega} \rightarrow[0 ; 1]$ is called a mass function that defines how belief is distributed across the frame, if the function satisfies the following conditions, for hypotheses A:

$$
\begin{gathered}
m(\Phi)=0 \\
\sum_{A \in \theta} m(A)=1
\end{gathered}
$$

Based on these conditions, belief from an evidence source cannot be assigned to an empty or null hypothesis, and belief from the evidence source across the possible hypotheses (including combinations of hypotheses) must sum to 1 , similar to probability theory. The least informative evidence (uncertainty) is the assignment of mass to a hypothesis containing all the elements $\left\{h_{1}, h_{2}, \ldots h_{\mathrm{n}}\right\}$, because this evidence does not commit to any particular hypothesis. This uncertainty is denoted by the symbol $\theta$

A crucial part of the process of assessing evidence is the ability to fuse evidence from multiple sources. In Dempster-Shafer theory, the combination of evidence from two different independent sources is accomplished by Dempster's combination rule:

$$
m_{12}(\mathrm{~A})=\frac{\sum_{X \cap Y=A} m_{1} \cdot m_{1}(X) \cdot m_{2}(Y)}{1-\sum_{X \cap Y=\varnothing} m_{1}(X) \cdot m_{2}(Y)}
$$

where $m_{12}(A)$ is the combined belief for a given hypothesis $\mathrm{A}$, and $\mathrm{X}$ and $\mathrm{Y}$ represent all possible subsets of the frame. The numerator in equation (3) represents evidence for hypotheses whose intersection is the exact hypothesis of interest, A. i.e. the agreement across the two sources about the hypothesis A. This denominator, $1-K$ is a normalisation factor, where $K$ is a conflict factor representing all combined evidence that does not match the hypothesis of interest, A. The value of conflict, $K$, when combining evidence is indicative of the level of disagreement amongst the sources of their belief in hypothesis A. Dempster's rule can be considered as a strict AND operation of the evidence sources [22].

\subsection{DS evidence fusion issues}

During our work on situation recognition [23] [3], we have noted three particular problems for when we apply Dempster's rule of combination to infer situations from sensor data:

1. Zadeh's paradox: Zadeh's paradox is a well-documented problem with Dempster's rule of combination [21]. Zadeh highlighted the fact that when sources in 
high conflict are combined using Dempster-Shafer rule, the results can be completely counter intuitive.

2. Single Sensor Dominance: A second problem that has gained far less attention in the literature is the potential dominance of a single sensor. Murphy [8] described how a single disagreeing sensor can overrule multiple other agreeing sensors in the fusion process. A categorical belief function is where all belief is assigned to one hypothesis in a frame [24]. For example, if five sensors are used to determine the location of a user in the house, a single categorical sensor that assigns all of its belief to a contradictory option will negate the evidence from the other four sensors. We suggest that this is particularly problematic for binary sensors which are increasingly being used in smart home deployments. Binary sensors typically have small frames of discernment, with just three hypotheses: \{on, off, $\theta$ \}. Unless discounted, they will categorically assign all of their belief to the 'on' or 'off' states. A single malfunctioning binary sensor can in theory therefore overrule evidence from other correct binary sensors during the fusion process, unless its off state is assigned as evidence of uncertainty. A more intuitive result would be to allow the agreeing sensors to 'win' but to represent the disagreeing sensors' evidence as conflict.

3. Evidence Spread Over Time: A third problem that we have observed [3] occurs when sensors' evidence of a higher-level state is spread over time. For example, the detection of a breakfast activity is the sequence of the triggering of a fridge sensor, then a kettle sensor, then the toaster sensor and so forth. At any point in time, only one of the sensors may be 'on', so fusion of all the sensor values at any point in time may result in the 'on' sensor evidence being lost. The fusion rule should capture that some evidence of the situation was observed even though it has been greatly contradicted by sensors that are off. It should not be wiped out by the overruling of the contradictory sensors, as will occur with Dempster's rule of combination.

\subsection{Alternative evidence fusions approaches}

Dempster's rule of combination was the core fusion mechanism provided as part of the original Dempster-Shafer theory. The rule has been enhanced or changed by researchers in order to cater for specific applications of Dempster-Shafer theory because it is not suitable in all fusion scenarios, such as the work of Yager [6], Hong et al [20], Dubois and Prade [7], Smets [25] and Murphy [8]. To narrow down our focus, we examine the variations that address the particular fusion issues we have discussed in Section 3.2

\section{Murphy's combination rule}

Murphy's [8] alternative rule of combination eliminates the dominance of a single sensor and allows contradictory evidence to be preserved to some degree. Evidence is averaged prior to combining it using Dempster's rule of combination. Formally, if 
there are $n$ sources of evidence, we use equation (3) to combine the weighted averages of the masses $n-1$ times. Evidence for each hypothesis, $h$, from $n$ sources is summed, and averaged across all evidence sources. This eliminates the dominance of a single sensor by reducing its contribution according to the number of sources. Use of Murphy's combination rule will also eliminate Zadeh's paradox because the evidence is averaged prior to combination.

\section{Averaging rule}

Shafer [9] combined belief functions by averaging all the evidence for each hypothesis (instead of the combination rule), as follows:

$$
M(A)=\frac{1}{n}\left(M_{1}(A)+\ldots+M_{n}(A)\right)
$$

Averaging can be used to eliminate the influence of any strongly conflicting single belief [22] so would cater for both single sensor dominance and Zadeh's paradox. The use of averaging provides an accurate record of contributing beliefs because no belief is lost, but it lacks convergence. Both Dempster's and Murphy's rule allows evidence from sources that are in agreement to reinforce each other, and disagreeing evidence to be dropped. In contrast, averaging does not increase the measure of belief in the dominant subset but provides a less conclusive picture because conflict is not normalised out. However, it is simpler to compute with fewer calculations. We anticipate that averaging will be useful to counteract the expected problem of conflicting sensors in binary sensors.

\section{OR Combination rule}

Hong et al [20] selected belief from two evidence sources by selecting the maximum belief. From our own work, we can see that this may apply well where a set of separate binary sensors are used for evidence of a situation. For example, the situation of 'preparing breakfast' may be detected by the kettle sensor firing or the toaster sensor. Mathematically, the highest mass associated with hypothesis A is selected from the evidence sources:

$$
M(A)=\max \left(m_{1}(A) . . m_{n}(A)\right)
$$

This may be useful for task-driven situations where the sensors are triggering over a period of time. In theory, only a single piece of evidence needs to be triggered in order to detect the situation using the OR fusion scenario.

\section{Temporal knowledge in fusion rules}

Temporal knowledge is a natural human way to reason about current activities or situations. For example, when assessing the current activity of a person at home, the 
time of day may determine whether they are preparing breakfast or dinner; the length of time they spent in the kitchen may help us decide whether they were preparing a meal or just getting a drink, and so on. Time durations of situations, sequential patterns in which situations occur and discernible patterns over time are examples of temporal knowledge that can improve our ability to recognise which situation(s) is occurring.

In [3], we set out an approach to incorporating time into situation recognition, using evidence theory. To recap, we extended transitory evidence to increase the knowledge of our sensor datasets as follows.

We define transitory evidence [3] as evidence that does not last for the full duration of a situation. For example, during the situation of 'preparing breaking', the kettle sensor may fire briefly just one, even though the situation may be in progress over a number of minutes. During the inference process, when evidence for that situation is detected, the duration for that situation is triggered to start. Looking at the situation of 'preparing dinner' in figure Fig. 1, when any of the groceries cupboard, fridge, freezer, pans cupboard or plates cupboard sensors are fired, the reasoning system will 'start' the dinner activity. The lifetime of the triggered sensor evidence for that activity will be extended to last for the remaining duration stored for that situation. As inference continues over time, the lifetime of any further evidence for the situation will be extended for the duration that is left of the situation (i.e. situation duration elapsed time). Once the full duration of the situation is reached, the evidence will expire. By extending the lifetime of the evidence, at any point in time, the evidence sources can be fused as if they are co-occurring.

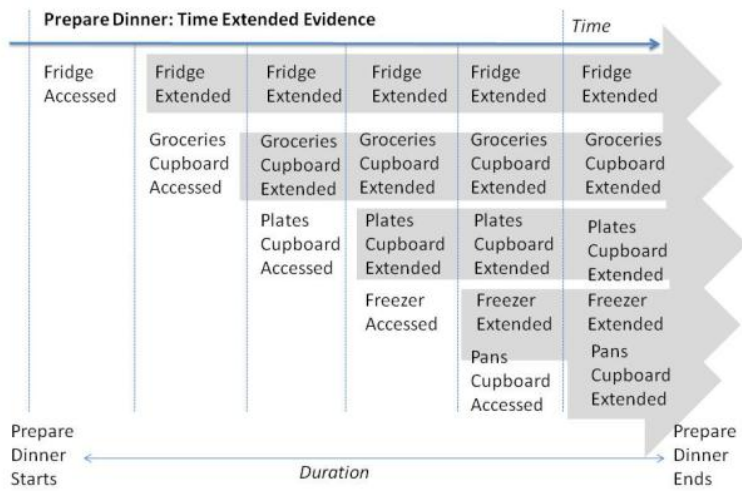

Fig. 1. Time extension of evidence for 'preparing dinner' situation 


\section{Sensor Datasets}

In our work, we have used two annotated data sets containing data from sensors, allowing us to apply our various evidence approaches to situation recognition using the sensor data. Using the data sets, we will measure situation recognition success using Dempster's original rule of combination, Murphy's rule, Averaging rule and OR combination rule. In addition, we will do each of these using transitory and time extended evidence.

\subsection{Van Kasteren DataSet}

This data set originates from the intelligent autonomous systems group in University of Amsterdam [16]. The data is over a 28 day period using 14 digital sensors were installed in the house. The sensors are installed on the hall-toilet door, hall-bathroom door, hall-bedroom door, front door, microwave, fridge, freezer, washing machine and each of the cups/plates/pans cupboards. When a sensor is fired, it outputs a value 1 as its reading in the sensor output file.

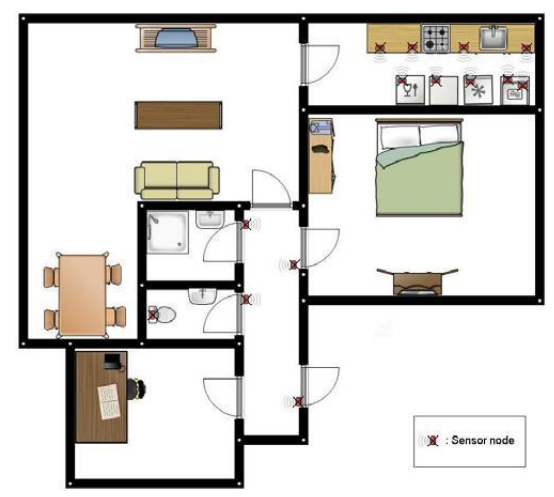

Fig. 2. Layout of sensors in van Kasteren house floor plan [109]

Seven situations (termed 'activities') are annotated by the occupant of the house: 1) leave house; 2) use toilet; 3) take shower; 4) go to bed; 5) prepare breakfast; 6) prepare dinner; 7) get drink as shown in Fig. 2.

We timesliced the data to 1 minute timeslices, so that the sensor values are known at minute variations. The full data preparation, and domain knowledge attached to sensor readings is described in our previous work on temporal evidence theory in [3]. For the purposes of this work, it is worth emphasising that the sensor readings in Van Kasteren are from binary sensors, and generate transitory evidence - evidence which does not last for the full duration of the situation. 


\subsection{CASL data set}

Our second data set, termed CASL, was generated in the Complex and Adaptive Systems (CASL) research laboratory of University College Dublin. We built our own infrastructure to capture the following data about a person in our research lab environment: their computer activity, their calendar entries, and their physical location in the building. Describing each sensor in turn:

- The computer activity sensor runs on the participant's desktop PC and monitors the rate of key presses and mouse clicks, along with the length of time since the last activity. The data from this sensor is used to indicate whether the user is 'active' or 'inactive' at their desktop. This data set is described and used in [2]. It is available to download at www.comp.dit.ie/smckeever/research.html

- The calendar sensor collects information about the user's scheduled Google calendar events, including meeting schedule state, start time and end time. The data from this sensor indicates whether the user has a meeting or not for the current time. The context values used are 'meeting' and 'no meeting'. The sensor mass function assigns its belief to 'meeting' if there is a meeting scheduled in the diary for the time in question, and assigns belief to 'no meeting' if the diary is empty.

- For Location sensing, we have a Ubisense system deployment on the third and fourth floor of our research lab. Ubisense is a tag-based 3-D location tracking system. It provides an $\mathrm{X}, \mathrm{Y}$ and $\mathrm{Z}$ coordinate, based on the number of metres from an origin point - in our case the bottom corner of the third floor in CASL. Ubisense covers areas on the third and fourth floors in the CASL building, using 30 wallmounted sensors. Ubisense tracks a location tag belonging to the user, providing co-ordinate readings for the location tag when the tag is moved. The participant gathered a data set over a 5-day period. For situation detection, as previously stated, we are interested in four particular locations: desk, cafe, meeting room and all other locations. Therefore, the context values are user desk, cafe, meeting room, other. The frame for the sensor mass functions contains the singleton elements: \{desk, cafe, meetingRoom, other\} and all possible combinations of the singletons: $\left\{\right.$ desk $^{\wedge}$ cafe; desk $^{\wedge}$ cafe $^{\wedge}$ meeting,,..$\left.\theta\right\}$ : In practice, most of the combined elements are never used.

Six situations were annotated: (1) busy at computer, (2) busy reading at desk, (3) coffee break, (4) lunch break, (5) informal break, and (6) meeting. Each of these is detectable from the combination of the three sensor systems in our infrastructure. At any point in time, the participant can only be engaged in one of these situations, but is always engaged in one of the situations.

\section{$5 \quad$ Empirical Comparison of Fusion Rules}

The purpose of our experiment is to compare the situation recognition rates of various evidential fusion rules when used against our two data sets. We use the standard 
$f$-Measure metric, the weighted mean of precision and recall as our measure of comparison as also used in [3]. The full evidential reasoning for the van Kasteren dataset is described in [3] and for the CASL dataset in [23].

\subsection{Results from van Kasteren Data set}

Table 1. shows a comparison of situation recognition accuracies when each of the four rules is used with van Kasteren's data set. Dempster's rule is least accurate when transitory sensor evidence is used. We can anticipate this because Dempster's rule will allow a single sensor to overrule other sensors. Therefore, unless all sensors are fired at the same time in one time slice, the evidence from any firing sensors will be lost or allocated to uncertainty. When evidence from the sensors is extended for the duration of the situation, Dempster's rule improves greatly, as the problem of the single sensor dominance no longer exists.

Table 1. Recognition accuracies (f-measure) for four fusion rules on van Kastersen's data set.

\begin{tabular}{|l|c|c|c|c|}
\hline Fusion Rule: & OR & Averaging & Dempster & Murphy \\
\hline $\begin{array}{l}\text { Situation recognition with } \\
\text { transitory evidence }\end{array}$ & $\mathbf{0 . 5 7}$ & 0.54 & 0.48 & 0.55 \\
\hline $\begin{array}{l}\text { Situation recognition with } \\
\text { time extended evidence }\end{array}$ & 0.66 & 0.65 & 0.60 & $\mathbf{0 . 6 8}$ \\
\hline
\end{tabular}

Murphy's rule performs better than Dempster's in both cases. The agreeing sensors reinforce each other. In the case of transitory evidence, the categorical off sensors do not negate the evidence of the on sensors.

The averaging rule produces results that are almost as good as Murphy's rule. The averaging rule uses less computation but it does not provide any measure of conflict. At present, we do not use the conflict metric in our inference process so averaging may provide a faster alternative to evidence fusion, if the capture of a conflict measure is not an issue.

Interestingly, the 'OR' fusion rule performs best when used with transitory evidence. Only the 'OR' rule disregards co-occurrence of evidence, because it only relies on single pieces of evidence. The 'OR' approach also has issues distinguishing situations if they share sensors. For example, if a cup is used, and this is equally evidential of breakfast and drink, the system cannot distinguish which situation is occurring. When evidence is time-extended, all approaches, including the 'OR' fusion approach, benefit from longer durations of evidence, as previously discuss in [3]. 


\subsection{Results from CASL dataset}

We run a similar analysis on the CASL data set, comparing results for fusion using averaging, Dempster's rule and Murphy's rule. We excluded the 'OR' combination rule because all evidence is co-occurring in the CASL situations so there is no theoretical benefit in using 'OR' fusion.

Looking at Table 2, in the CASL data set, Demptser's rule of combination performs slightly better than Murphy's rule. Unlike van Kastersen's data set, all evidence is continuous so the problem of single sensors being 'off' and overruling other firing sensors is not an issue. The averaging rule performs relatively poorly. In the CASL data set, evidence is frequently applied to combinations of situations (as opposed to the van Kasteren data set where evidence was applied to single situations). For example, if the computer activity sensor is 'inactive', this is indicative of any of the situations 'busy at desk', 'coffee break', 'lunch break', 'informal break' or 'meeting'. When averaging is used, the evidence is simply divided up amongst the elements prior to averaging. In Dempster's rule and Murphy's rule, agreeing evidence is merged so combined evidence converges more distinctly towards situations that have further evidence. As a result, the averaging rule results are more successful in van Kastersen's data set than in the CASL data set.

Table 2. Situation recognition (f-measure) for three fusion rules on the CASL data set.

\begin{tabular}{|l|c|c|c|}
\hline Fusion Rule & Averaging & Dempster & Murphy \\
\hline Situation recognition & 0.5 & $\mathbf{0 . 6 2}$ & 0.60 \\
\hline
\end{tabular}

\subsection{Discussion}

The results confirm that the choice of evidential fusion rule has an impact on the success of inferring the correct situations on our data sets. From our analysis, we emphasise the following points for practitioners using evidence theory for analysing sensor data.

1. If the environment is generating sensors readings that are occurring over time, Dempster's rule of combination is useful for evidence that is cooccurring. In a monitored environment, multiple sensors may be used to detect a particular activity. If these sensors do not fire concurrently, Dempster's rule should be used with care. At any time, if most sensors associated with a situation are off, they will be evidential of the activity not occurring or uncertainty and may therefore give a misleading result.

2. In an environment where multiple sensors are used to detect a situation, and these sensors give short burst readings, consider the use of the OR fusion 
rule as a simple way to determine that if any sensor for a situation is on, the situation may be occurring.

3. The frame of discernment and allocation of evidence towards each element in the frame are a key consideration when choosing the evidence fusion in rule. We saw that for the CASL data set, a sensor may be evidential of more than one situation - in which case, the use of the averaging rule is weakened for reasons previously stated.

4. Finally, we note that computational complexity of the fusion rules is another consideration. For both Murphy's and Dempster Rule of Combination, evidence can be applied for any combination of hypotheses in a frame, so the number of permutations for $n$ hypotheses in a frame can be as high as the power set, $2^{n}$. But in reality, all combinations of hypotheses may never be used and there is no need to always build all the possible values of belief. There are many cases where the knowledge is very simple and where there are very few non-null masses - making the belief function computation lighter than its competitors [32]. In addition, we noted that the simple averaging rule achieved comparable results with Murphy's decision rule on van Kastersen's data set. The averaging rule works well if applied to evidence that support single hypotheses only in a frame. Another way to reduce computational effort is to limit calculations to active parts of the environment. For example, using an example from Hong et al.'s [23] smart home, a motion sensor may detect motion in the kitchen. At that point in time, situation beliefs will only be calculated for kitchen-based situations.

\section{Conclusions}

In this work, we have compared a number of variations to the Dempster rule of combination, based on resolving specific issues encountered during previous work: Zaheh's paradox, single sensor dominance and transitory evidence. Our results for our first data set show that Murphy's rule produces the highest situation recognition rate when used with time extended evidence. When using binary trigger sensors, the simple OR rule produces the best results. In our second data set, where all sensors are continuously generating evidence, Murphy's rule is no longer needed to negate a single sensor dominating, and Dempster's rule performs best. Most importantly, to understand these results, a deep knowledge of the sensor data values and their use for inference over time is required in order to select the most suitable fusion rule. For example, Murphy's alternative fusion rule [8] negates the effect of a single dominant sensor overruling the evidence-based decision. While our work supports this observation, we note that this is applicable if all evidence is co-occurring. It is either less or not applicable if sensor readings for a situation are triggering over time and not occurring simultaneously The selection of suitable frames of discernments, either singletons or combined will also influence the outcomes. 


\section{$7 \quad$ References}

\section{Bibliography}

[1] Simon Dobson and Paddy Nixon, "Whole-Systems Programming of Adaptive Ambient Intelligence," Universal Access in HCI, AmbientInteraction, vol. 4555/2007, pp. 73-81, 2007.

[2] Susan McKeever, Juan Ye, Lorcan Coyle, and Simon Dobson, "A Context Quality Model to Support Transparent Reasoning with Uncertain Context," in Lecture Notes in Computer Science, vol. 5786/2009, 2009, pp. 65-75.

[3] Susan McKeever, Juan Ye, Lorcan Coyle, and Simon Dobson, "Activity recognition using temporal evidence theory," Journal of Ambient Intelligence and Smart Environments, vol. 2, 2010.

[4] Huadong Wu, Mel Siegel, and Rainer Stiefelhagen, "Sensor fusion using Dempster-Shafer theory," in in Proceedings of IEEE Instrumentation and Measurement Technology Conference, vol. 1, 2002, pp. 7--12.

[5] Daqiang Zhang, Minyi Guo, Jingyu Zhou, Dazhou Kang, and Jiannong Cao, "Context reasoning using extended evidence theory in pervasive computing environments," Future Gener. Comput. Syst., vol. 26, pp. 207--216, 2010.

[6] Ronald R Yager, "On the Dempster-Shafer framework and new combination rules," Inf. Sci., vol. 41, pp. 93--137, 1987.

[7] Didler Dubois and Henri Prade, "Representation and combination of uncertainty with belief functions and possibility measures," Computational Intelligence, vol. 4, pp. 244-264, 1988.

[8] Catherine K Murphy, "Combining belief functions when evidence conflicts," Decis. Support Syst., vol. 29, pp. 1--9, 2000.

[9] Glenn Shafer, A mathematical theory of evidence.: Princeton Unverisity Press, 1976.

[10] P Korpipaa, J Mantyjarvi, J Kela, H Keranen, and E J Malm, "Managing context information in mobile devices," Pervasive Computing, IEEE, vol. 2, pp. 42-51, July-Sept. 2003.

[11] Emmanual Tapia, Stephen Intille, and Kent Larson, "Activity Recognition in the Home Using Simple and Ubiquitous Sensors," , vol. 3001/2004, 2004, pp. 158175.

[12] A Ranganathan, J Al-Muhtadi, and R H Campbell, "Reasoning about uncertain contexts in pervasive computing environments," Pervasive Computing, IEEE, vol. 3, pp. 62-70, April-June 2004.

[13] Tao Gu, Hung K Pung, and Da Q Zhang, "A Bayesian approach for dealing with uncertain contexts," , 2004, pp. 205--210.

[14] Brian Clarkson, Alex Pentland, and Kenji Mase, "Recognizing User Context via Wearable Sensors," Wearable Computers, IEEE International Symposium, p. 69, 
2000.

[15] Driss Choujaa and Naranker Dulay, "Activity Inference through Sequence Alignment," , 2009, pp. 19--36.

[16] Tim van Kasteren, Athanasios Noulas, Gwenn Englebienne, and Ben Krã $q$ se, "Accurate activity recognition in a home setting," , 2008, pp. 1--9.

[17] Huadong Wu, "Sensor fusion using dempster shafer theory," phdthesis 2003.

[18] Huadong Wu, Mel Siegel, and Rainer Stiefelhagen, "Sensor fusion using Dempster-Shafer theory," , vol. 1, 2002, pp. 7--12.

[19] Xin Hong et al., "Uncertain Information Management for ADL Monitoring in Smart Homes," Intelligent Patient Management, vol. 189/2009, pp. 315-332, 2009.

[20] Xin Hong et al., "Evidential fusion of sensor data for activity recognition in smart homes," Pervasive and Mobile Computing, vol. 5, pp. 236-252, 2009.

[21] Lotfi A Zadeh, "A Simple View of the Dempster-Shafer Theory of Evidence and its Implication for the Rule of Combination," AI Magazine, vol. 7, pp. 85--90, 1986.

[22] Karl Sentz and Scott Ferson, "Combination of Evidence in Dempster Shafer Theory," techreport 2002.

[23] Susan McKeever, "PhD Thesis: Recognising Situations using extended Dempster-Shafer Theory," phdthesis 2011.

[24] Weiru Liu, "Analyzing the degree of conflict among belief functions," Artif. Intell., vol. 170, pp. 909--924, 2006.

[25] Phillippe Smets and Robert Kennes, "The Transfer Belief Model," Symbolic and Quantitative Approaches to Uncertainty, pp. 91-96, 1991. 